\title{
Editorial
}

\section{Numerical Methods for Solving Variational Inequalities and Complementarity Problems}

\author{
Abdellah Bnouhachem ${ }^{1}$ and $\mathrm{Min}_{\mathrm{Li}}{ }^{\mathbf{2}}$ \\ ${ }^{1}$ Ibn Zohr University, BP 1136, ENSA, Agadir, Morocco \\ ${ }^{2}$ School of Economics and Management, Southeast University, Nanjing 210096, China \\ Correspondence should be addressed to Abdellah Bnouhachem, babedallah@yahoo.com \\ Received 25 June 2012; Accepted 25 June 2012 \\ Copyright (C) 2012 A. Bnouhachem and M. Li. This is an open access article distributed under \\ the Creative Commons Attribution License, which permits unrestricted use, distribution, and \\ reproduction in any medium, provided the original work is properly cited.
}

This special issue focuses on algorithmic approaches to solve variational inequality problems. It includes 5 papers selected after a peer revision. We hope that the readers will find in these papers stimulating ideas and useful results. The summaries of the 5 papers in this issue are listed as follow:

The paper by $\mathrm{X}$. Fu et al., proposes an asymmetric proximal decomposition method to solve a wide variety separable problems by combining the ideas of proximal point algorithm and augmented Lagrangian method. Numerical experiments are employed to show the advantage of the proposed method.

The paper by N. K. Mahato and C. Nahak has been aimed to theoretically study the existence of solutions for variational-like inequality problems under a new concept relaxed $(\rho-\theta)-\eta$-invariant pseudomonotone maps in reflexive Banach spaces.

The paper by Min Li considers the split feasibility problem, which is a special case of the multiple-sets split feasibility problem. With some new strategies for determining the optimal step length, this paper improves some known halfspace-relaxation projection methods for solving the split feasibility problem. Some preliminary computational results are given to illustrate the efficiency and implementation of the proposed method.

Based on the auxiliary principal technique and arguments from generalized convexity, the paper by $\mathrm{H}$. Mahdioui and O. Chadli studies the existence and the algorithmic aspect of a system of generalized mixed equilibrium problems (SGMEP) involving variationallike inequalities in Banach spaces. A new existence theorem for the auxiliary problem is established; this leads to generate an algorithm that converges strongly to a solution of SGMEP under weaker assumptions.

The paper by M. H. Xu and H. Shao considers the matrix nearness problem. Based on the relationship between the matrix nearness problem and the linear variational inequality, 
a projection and contraction method is presented for solving the matrix nearness problem. Numerical results show that the method suggested in this paper has a good performance.

\section{Acknowledgment}

Guest editors would like to take this opportunity to express their sincere thanks to all the authors for their contributions to this special issue and the reviewers for their comments and suggestions.

Abdellah Bnouhachem

Min $\mathrm{Li}$ 


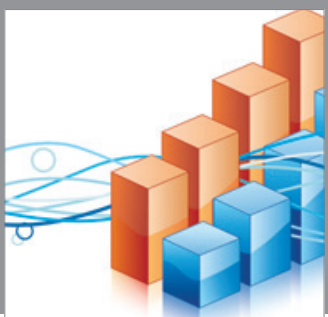

Advances in

Operations Research

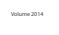

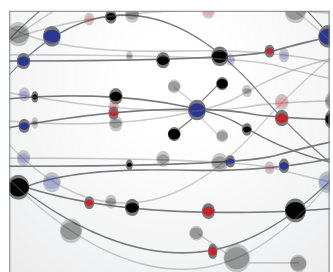

\section{The Scientific} World Journal
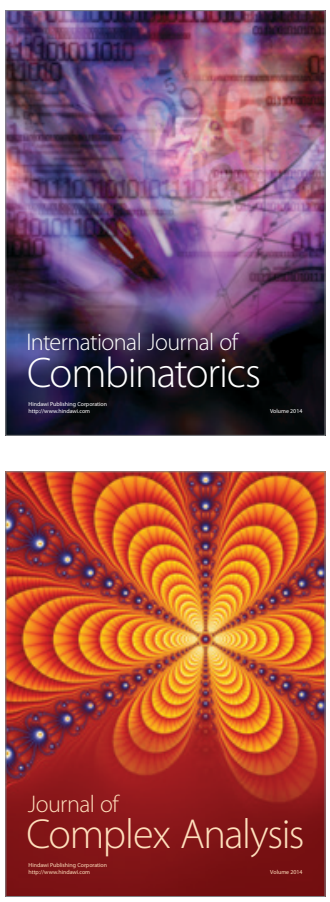

International Journal of

Mathematics and

Mathematical

Sciences
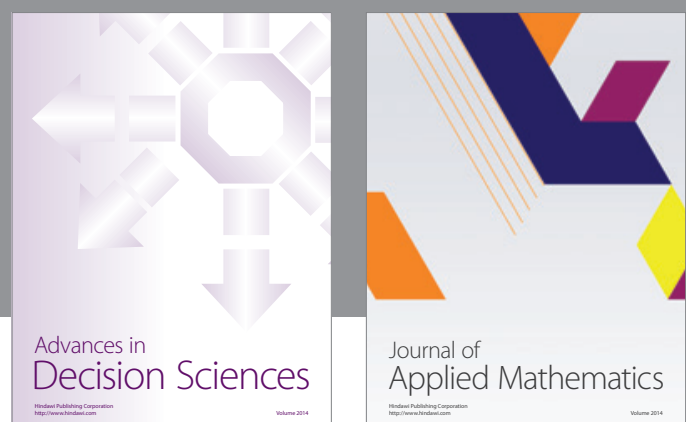

Journal of

Applied Mathematics
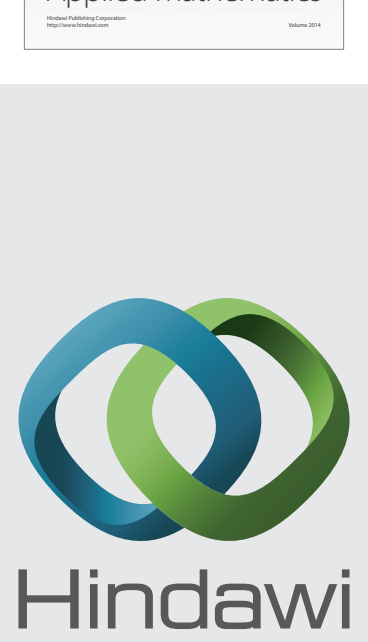

Submit your manuscripts at http://www.hindawi.com
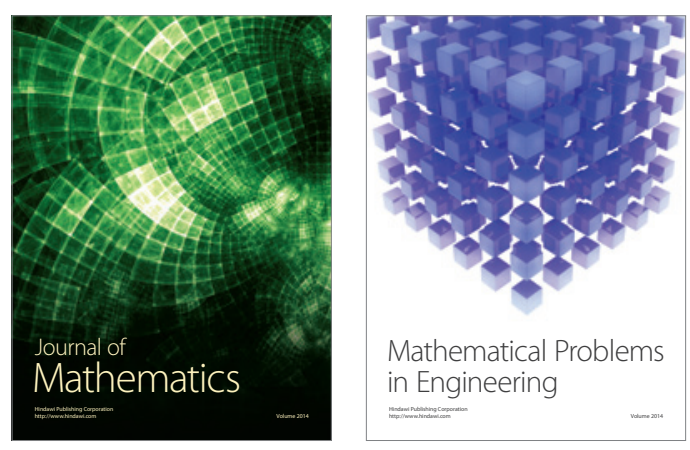

Mathematical Problems in Engineering
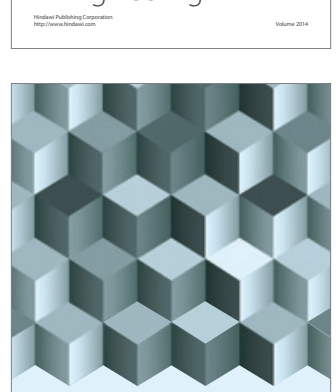

Journal of

Function Spaces
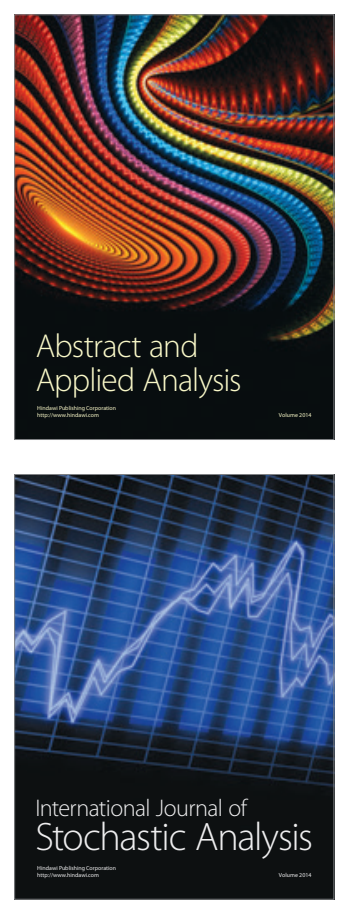

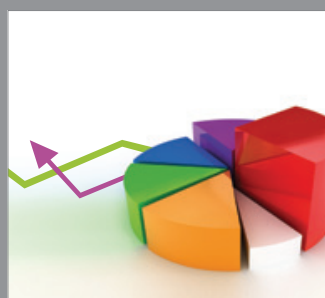

ournal of

Probability and Statistics

Promensencen
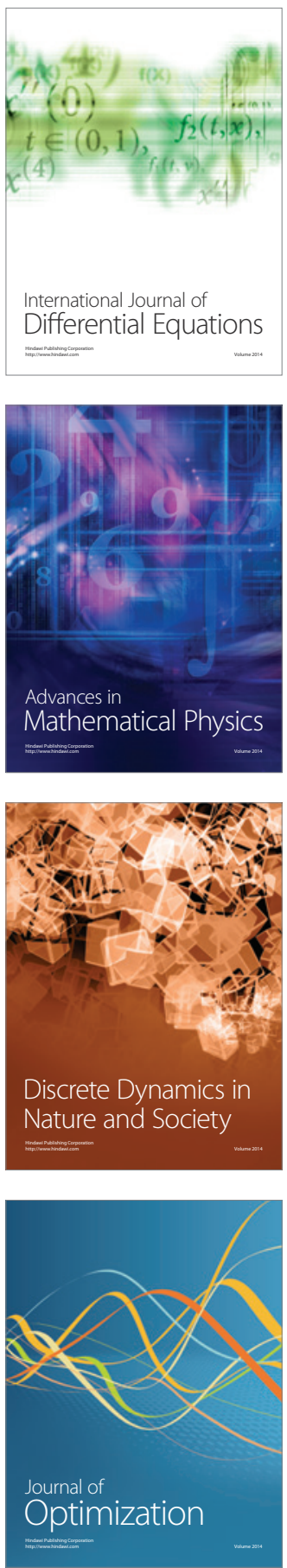